\title{
BMJ Open Tackling statin intolerance with n-of-1 trials (TaSINI) in primary care: protocol for a feasibility randomised trial to increase statin adherence
}

Kate Tudor, ${ }^{1}$ Jenny Brooks, ${ }^{1}$ Jeremy Howick, ${ }^{2}$ Robin Fox, ${ }^{3}$ Paul Aveyard (D) ${ }^{1,4}$

To cite: Tudor K, Brooks J, Howick J, et al. Tackling statin intolerance with n-of1 trials (TaSINI) in primary care: protocol for a feasibility randomised trial to increase statin adherence. BMJ Open 2020;10:e033070. doi:10.1136/ bmjopen-2019-033070

- Prepublication history for this paper is available online. To view these files, please visit the journal online (http://dx.doi. org/10.1136/bmjopen-2019033070).

Received 22 July 2019 Revised 18 December 2019 Accepted 20 December 2019

Check for updates

(c) Author(s) (or their employer(s)) 2020. Re-use permitted under CC BY-NC. No commercial re-use. See rights and permissions. Published by BMJ.

${ }^{1}$ Nuffield Department of Primary Care Health Sciences, University of Oxford, Oxford, UK

${ }^{2}$ Faculty of Philosophy, University of Oxford, Oxford, UK

${ }^{3}$ Bicester Health Centre,

Bicester, UK

${ }^{4}$ Primary Care Health Sciences, University of Oxford, Oxford, UK

Correspondence to

Dr Kate Tudor;

kate.tudor@phc.ox.ac.uk

\section{ABSTRACT}

Introduction Statins reduce the incidence of cardiovascular disease (CVD) and cause few adverse effects. Half of patients prescribed statins discontinue treatment due to perceived intolerance. Placebo-controlled (blinded) n-of-1 trials have shown people with perceived intolerance that the statin does not cause adverse events and most resume treatment. However, blinded n-of-1 trials are impractical to deliver in routine practice. Tackling Statin Intolerance using $\mathrm{n}$-of- 1 trials (TaSINI) will test the feasibility of a general practitioner (GP)-delivered behavioural intervention endorsing an unblinded $n-0 f-1$ trial to increase adherence to statins relative to usual care. Methods and analysis TaSINI is a feasibility randomised controlled trial with a nested qualitative substudy. Ninety primary care patients who have discontinued statins due to intolerance or refused treatment will be randomised to an unblinded n-of-1 trial, a blinded n-of-1 trial (positive control) or usual care (negative control). Participants randomised to usual care will be advised to take statin therapy to prevent CVD. In both n-of-1 trial arms, GPs will deliver a behaviourally informed intervention that accessibly explains the benefits of statins, the prevalence of adverse effects and endorse the benefit of experimenting with medication. Participants will alternate between 4 weeks of medication and no medication (unblinded arm) or randomly sorted active and placebo (blinded arm) and will record adherence, symptoms and symptom attributions throughout. After 6 months, GPs will feedback symptom data during active/inactive treatment periods. All participants will be asked if they would like to initiate statin treatment. Measures of feasibility will be met if $4 \%$ of invited patients enrol, $50 \%$ of participants randomised to $\mathrm{n}$-of- 1 trials engage with the experiment and $25 \%$ more participants initiate statin in the unblinded n-of- 1 arm than in usual care.

Ethics and dissemination This study has been granted ethical approval by North of Scotland Research Ethics Service. The results will be written up for publication and show whether to progress to an effectiveness trial where the primary outcome would be differences in low-density lipoprotein concentration.

\section{INTRODUCTION}

Statins reduce the incidence of fatal and non-fatal cardiovascular disease (CVD), and

\section{Strengths and limitations of this study}

This trial will test a new approach for general practitioners and patients to determine the cause of adverse effects during statin use and allow patients to make an evidence-based decision on whether to start statin therapy or not.

- The consent procedure will result in the inclusion of hard- to-reach patients who have previously experienced intolerable statin adverse effects, who may otherwise have declined to participate in a trial that may involve statin use.

- Qualitative and quantitative analyses will assess the feasibility of the intervention, informing the development of an effectiveness trial.

- Some people may have adverse reactions to statins that do not resolve with a 3-week washout in the $\mathrm{n}$-of-1 design and this approach will not be helpful for them.

- The study will not demonstrate the clinical effectiveness of this approach, and a definitive trial will be required to test whether this intervention can lead to reductions in cardiovascular risk.

reduce all-cause mortality. ${ }^{12}$ Severe adverse reactions include the development of type- 2 diabetes, rhabdomyolysis and haemorrhagic stroke, however these are extremely rare. ${ }^{34}$ Evidence from non-randomised, nonblinded, observational studies suggest statins are related to muscle pain (in the absence of myopathy), ${ }^{56}$ and there has been widespread reporting of such findings in the lay media. ${ }^{7}$ However, randomised controlled trials (RCTs) suggest statins are well-tolerated in most users and have not found evidence that statins cause muscle pain, but this may be because participants with muscle pain drop out of treatment during the run-in phase prior to randomisation. ${ }^{18}$

Clinical trials and national guidelines provide reassurance of the benefits, safety and tolerability of statins $;{ }^{9}$ however, about half of new starters discontinue the medication 
within the first year. ${ }^{10}$ Discontinuation is commonly a result of intolerable adverse effects, primarily muscle pain, ${ }^{11}$ and evidence indicates that the prevalence of statin discontinuation increases after periods of increased media coverage that highlight these effects. ${ }^{7}$ One explanation for statin intolerance in routine practice is that patients misattribute their experience of adverse events from unrelated causes to the statin medication. A recent review of 14 RCTs tested the proportion of symptomatic adverse events in participants taking statin medication compared with placebo. ${ }^{1}$ Many of the adverse effects commonly attributed to statins, including muscle aches and myopathy, were no more prevalent in participants taking statins compared with placebo, suggesting participants were attributing unrelated to symptoms to both study medications. This misattribution may be exacerbated by the fact that musculoskeletal symptoms are common among the age group of patients who are prescribed statins. Another explanation is that patients who start taking statin medications are aware of the potential adverse effects, anticipate experiencing them and subsequently experience nocebo effects. ${ }^{12}$ Currently, clinicians do not have a diagnostic tool to inform patients whether the symptoms they are experiencing are caused by the statin, or something else.

N-of-1 trials use the key methodological elements of clinical trials to examine treatment effectiveness or adverse effects in individual participants and have been considered the pinnacle of the evidence hierarchy for making decision about treatment benefits versus harms for individuals. ${ }^{13}$ In randomised n-of- 1 trials, participants receive an active intervention (A) or control/inactive intervention (B), and they are randomised to a series of pairs that comprise a treatment sequence (eg, ABABAB, ABBABA). Participants can then be assessed both on and off medication and examine whether adverse effects are a result of the treatment or another cause. In a proof of concept trial, eight participants with presumed statin intolerance alternated between a randomised sequence of statin and placebo and reported daily pain symptoms. ${ }^{14}$ For each individual's n-of-1 trial, there was no clinically significant difference in pain symptoms while taking the statin compared with the placebo medication, and most patients resumed statin treatment full time. A larger scale study is currently ongoing which comprises a series of blinded, randomised n-of-1 trials in 200 primary care patients with perceived statin intolerance. ${ }^{15}$ This study aims to offer the opportunity for participants to determine whether the symptoms they experience are attributable to statins, by alternating between statin and placebo.

While blinded n-of- 1 trials are the gold-standard for determining whether symptoms are attributable to statins, it is not possible for clinicians to offer this approach in routine practice, due to the practical difficulties and expense of blinding medication. In the current trial, we aim to test whether an unblinded n-of-1 trial, where participants alternate between statins and no medication, can achieve the same outcome as a blinded n-of-1 trial. Using unblinded n-of-1 trials will reveal to participants whether they misattribute symptoms to statins. However, if the symptoms are 'nocebo' effects (ie, the result of expecting to experience symptoms while taking statins), they should still occur in an unblinded trial. Thus, we intend to use blinded n-of- 1 trials to act as a positive control condition to establish the true incidence of adverse effects caused by the statin. We will compare the outcome of both the blinded and unblinded n-of-1 interventions with routine care, where clinicians recommend statins to prevent CVD but do not offer the opportunity for patients to experiment with their treatment.

The aim of the Tackling Statin Intolerance using n-of-1 trials (TaSINI) study is to investigate the feasibility of a trial of a behavioural intervention delivered by a general practitioner (GP) endorsing an unblinded n-of-1 trial of statin medication to increase adherence to statin therapy relative to usual care. The objectives are to assess the feasibility of recruitment, agreement to try an n-of-1 study and the proportion of participants who agree to commence statins 6 months later. The TaSINI study will inform the sample size of a future trial, where the primary outcome would be differences in low-density lipoprotein (LDL) concentration, an outcome that would reduce the incidence of CVD. ${ }^{16}$

\section{METHOD}

\section{Trial design}

This feasibility study is an individually randomised, three-arm, controlled trial of a behavioural intervention to increase adherence to improve statin adherence. Participants will be adults with prior intolerance to statin medication or those who have previously refused a clinician's recommendation of statins. Participants will be enrolled for 6 months from receiving the intervention to final follow-up. Due to the nature of the intervention, it is not possible to blind participants, clinicians delivering the intervention or some of the study team to participants' allocation to the three treatments arms.

\section{Recruitment}

Participants will be recruited from several general practices. Practices will search their computerised records to identify people meeting the inclusion criteria and ensure that inviting them is appropriate and send an invitation letter. People interested in participating will contact the trial team to discuss participation and are offered an appointment and sent a participant information sheet (PIS), if appropriate. Potential participants may also be identified opportunistically by GPs in consultations. In this case, the GP will provide individuals with the invitation letter, and invite the patient to contact the study team for more information.

\section{Inclusion criteria}

Eligible patients include those who have previously discontinued statin treatment or have previously refused 
treatment following a recommendation from a clinician. Specific inclusion criteria are:

1 . Is $\geq 18$ years of age.

2. Requires statin therapy according to NICE guidelines and the GP thinks statins are indicated.

3. Has previously been prescribed/recommended statin treatment.

4. Has stopped/is considering stopping statin treatment/ or has not started statin treatment due to concerns about or experience of side effects.

5. Is willing and able to give informed consent for participation in the study and adhere to study procedures.

6. If on ezetimibe or other alternative to atorvastatin is willing to potentially cease said medication if randomised to one of the n-of- 1 experiments.

\section{Exclusion criteria}

Any patient who:
1. The GP thinks it is not indicated to recommence statins or the previous intolerance was severe enough to mean that recommencing statins may comprise significant risk to health.

2. Is unable to adhere to the study procedures through illness or infirmity.

3. Has any contraindications listed in the Summary of Product Characteristics for atorvastatin $20 \mathrm{mg}$ or placebo drug, including pregnancy.

4. Is participating in any other research study that might interact with the trial.

\section{Participant flow}

Figure 1 presents participant flow throughout the trial.

\section{Eligibility screening and informed consent}

Interested patients who contact the research team will be assessed over the phone to check additional eligibility

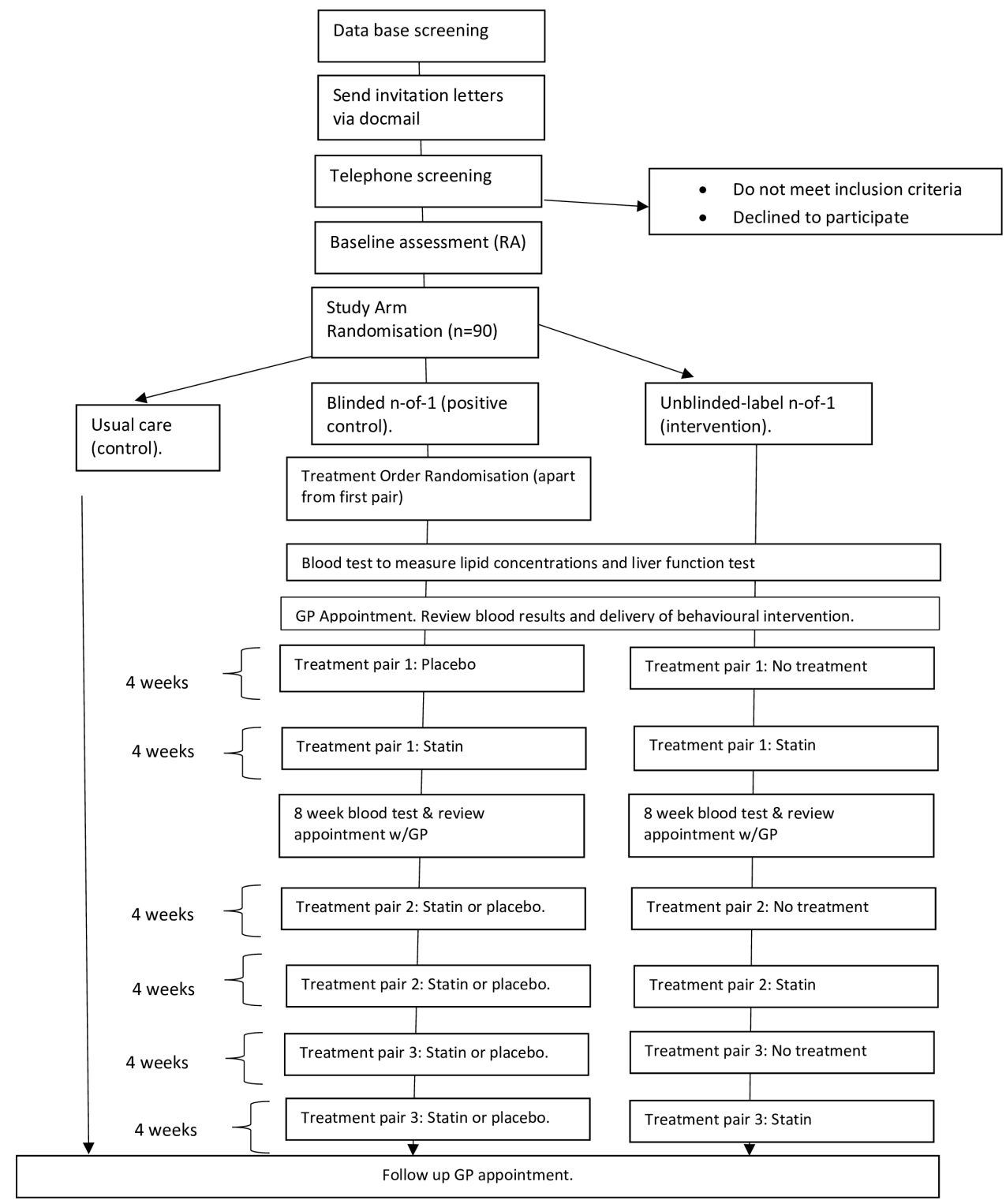

Figure 1 Participant flow. 
criteria. If potential participants meet the eligibility criteria they will be invited to attend a baseline visit with a researcher.

Refusing statins that are offered to prevent CVD can give rise to strong emotions about statins. In this trial, we are aiming to replicate normal practice in which patients would only hear about statins and behavioural experiment when meeting a doctor. Therefore, the PIS explains fully the nature of the trial but not the nature of the intervention nor the medication in question so that we can reflect clinical practice. Concealing these aspects avoids biased recruitment that could occur if the invitation letter or subsequent processes deterred those with strong negative feelings about statins.

\section{Study visits}

Participants allocated to the control arm will be informed that they will be contacted by a member of the trial team to attend an appointment with a GP to discuss ways to reduce their risk of CVD in approximately 6 months time.

Participants randomised to receive the n-of- 1 experiments will be invited to have a blood test at the practice and to attend a GP consultation shortly after. During the first GP visit, the GP will review the participants' blood results, deliver the behavioural intervention endorsing statin use and n-of- 1 experiments and provide participants with the appropriate medication (see Intervention section for more detail). After 8 weeks, participants in the n-of- 1 arms will be invited to have another blood test prior to a second GP consultation to assess effects on lipid profile and for rise in liver transaminases following UK guidelines. ${ }^{9}$ Here, the GP will review the blood results, provide the remaining trial medication and answer any questions the participant has about the n-of-1 trial.

\section{Online data collection}

For the last week of each 4-week treatment period, participants will be sent an email or text message and asked to complete an online daily questionnaire about adherence to the trial medication, their current symptoms and the attribution of these symptoms (see Measurements section for more details). Participants who are unable to access the internet will complete these on paper.

\section{Sample size}

The total number of participants recruited for this study will be 90 . As this is a feasibility study, it has not been powered to detect a statistically significant difference in CVD risk between the trial arms. The following progression criteria will determine whether to progress to a full trial:

1. That $4 \%$ of invited patients enrol into the trial. This is based on feasibility search of potentially eligible patients in one primary care practice.

2. That $50 \%$ of the enrolled participants randomised to the n-of- 1 arms accept the GP offer and attempt the nof-1 experiment after the first visit.
3. That the proportion of participants in the n-of- 1 arms who decide to restart statin therapy full time compared with the proportion who decide to restart in the control arm exceeds a difference of $25 \%$.

These feasibility outcomes are proportions (1 and 2) or differences in proportion (3) and we will be able to estimate these with the following precisions:

1. The proportion of invited patients who enrol in the trial $\pm 2 \%$.

2. The proportion of enrolled participants who accept GPs behavioural intervention $\pm 11 \%$.

3. Proportion of patients in the treatment conditions who decide to continue statin therapy compared with the proportion who decide to continue statin therapy in the control arm with a risk difference of $\pm 25 \%$.

These precisions are sufficient to make a stop-go decision for the main trial.

\section{Randomisation}

Randomisation of participants to trial arm

All eligible, consenting patients will be randomised to one of three trial arms: unblinded n-of-1 experiment (intervention), blinded n-of-1 experiment (positive control) or usual care (control), using a random permuted blocks of 5 and 10 . Allocation will be stratified by practice. An independent researcher will generate the set of sequences and assign participants to the trial arms using sequentially numbered sealed envelopes to ensure allocation concealment until trial arm is assigned by the researcher at the baseline visit.

\section{Treatment sequence in the $\mathrm{n}$-of- 1 trials}

In both n-of- 1 trial arms, the first treatment pair will be predetermined; participants will take no medication (unblinded) or placebo (blinded) for the first 4 weeks, and the statin for the second 4 weeks. This is predetermined to allow participants to have a liver function, creatine kinase and lipid test prior to the 8-week GP review visit, to ensure it is safe to continue statin treatment and to demonstrate the effect on lipids. For the second and third treatment pair, in the unblinded n-of-1 arm, participants will continue to alternate on and off medication in sequence (see table 1). In the blinded arm, the order of the statin or placebo will be randomly allocated within pairs according to a computer-generated list held by a pharmacist, who will have no contact with patients (see table 1). Participants will be blind to the treatment sequence throughout the n-of-1 trial. Clinicians will blind to the sequence of the second and third treatment pairs. Blinding will be maintained by use of identical-looking dispensing bottles and capsules in which statin or placebo pills will be compounded by the pharmacy. The GP delivering the intervention and the participant will be blind to the treatment order until the final study visit, when the research team will feedback the treatment order and corresponding symptoms that were experienced. 
Table 1 Non - randomised treatment sequence in the unblinded n-of-1 trial (top) and randomised treatment sequence in the blinded n-of- 1 trial (bottom)

\section{Treatment period}

$\begin{array}{llllll}1 & 2 & 3 & 4 & 5 & 6\end{array}$

\begin{tabular}{llllllll}
$\begin{array}{l}\text { Non-randomised treatment } \\
\text { sequence in the unblinded } \\
\text { n-of-1 trial }\end{array}$ & & & & & & \\
Participant/sequence 1 & Nil & s & Nil & s & Nil & s \\
Participant/sequence 2 & Nil & s & Nil & s & Nil & s \\
Participant/sequence 3 & Nil & s & Nil & s & Nil & s \\
Participant/sequence 4 & Nil & s & Nil & s & Nil & s \\
\hline
\end{tabular}

Treatment period

Predetermined

$\begin{array}{llllll}1 & 2 & 3 & 4 & 5 & 6\end{array}$

\begin{tabular}{lllllll}
$\begin{array}{l}\text { Randomised treatment } \\
\text { sequence in the } \\
\text { blinded } \mathrm{n} \text {-of-1 trial }\end{array}$ & & & & & & \\
Participant/sequence 1 & $\mathrm{p}$ & $\mathrm{s}$ & $\mathrm{s}$ & $\mathrm{p}$ & $\mathrm{s}$ & $\mathrm{p}$ \\
Participant/sequence 2 & $\mathrm{p}$ & $\mathrm{s}$ & $\mathrm{s}$ & $\mathrm{p}$ & $\mathrm{p}$ & $\mathrm{s}$ \\
Participant/sequence 3 & $\mathrm{p}$ & $\mathrm{s}$ & $\mathrm{p}$ & $\mathrm{s}$ & $\mathrm{s}$ & $\mathrm{p}$ \\
Participant/sequence 4 & $\mathrm{p}$ & $\mathrm{s}$ & $\mathrm{p}$ & $\mathrm{s}$ & $\mathrm{p}$ & $\mathrm{s}$ \\
\hline
\end{tabular}

\section{Interventions}

There are two arms where participants are supported to experiment with their medication (unblinded and blinded n-of-1 trials). In both arms, the GP will positively endorse the cardiovascular benefits of statin medication. Evidence suggests that patients may choose not to initiate (or discontinue) due to an insufficient explanation of statin necessity or physiological effect, or a belief that the medication will have reduced benefit over time. ${ }^{17-19}$ Both the blinded and unblinded n-of- 1 interventions were designed so the GP can explain this, and the explanation will be facilitated by an information booklet that presents the scientific evidence in an accessible way. The GP will explain to participants about the prevalence of adverse effects of statin in clinical trials versus routine practice. The GP will actively encourage patients to experiment with atorvastatin (20 mg) for a period of 4 weeks 'on' statin medication following 4 weeks 'off' statin medication. This process will be repeated three times (for a total of 6 months). The GP will explain that monitoring symptoms on each day and during the last week of each 4-week treatment period the GP will show whether or not the medication is causing side effects. A blood test and 8-week review appointment with the GP is incorporated as part of both n-of- 1 arms. This appointment requires the GP to review the blood test and to reassure the participant the statin medication is safe to continue. The difference between the two treatment arms is whether the participant is blinded to taking statin medication or not.

The intervention was developed following the principles of the person-based approach, which was used to enhance the acceptability, feasibility and effectiveness of the intervention. ${ }^{20}$ During intervention planning, we examined systematic reviews and qualitative studies of the predictors of discontinuation and non-adherence of statin therapy. Intervention planning was conducted within a multi-disciplinary team of primary care physicians, a psychologist and with patients' involvement. We met with patients who had discontinued a long-term medication due to side effects to refine the behavioural components of the GP intervention, booklet and selfexperimentation. Additionally, we surveyed 211 GPs to gain feedback on our intervention plans (see Patient, public and clinician involvement section for more details).

We used themes arising from the intervention planning stage to create guiding principles, comprising: (1) key intervention design objectives and (2) key distinctive features of the intervention to achieve objectives (see figure 2). The design of this intervention has been additionally informed by behavioural analysis, and identifies domains of the Behaviour Change Wheel $^{21}$ and the Theoretical Domains Framework ${ }^{22}$ to promote behaviour change. The intervention aims to allow participants to develop and sustain the psychological capability, social and physical opportunity and reflective motivation to change their medication-taking behaviour (see table 2 for a description of intervention components, primary messages and associated behaviour change techniques).

\section{Comparator}

Participants randomised to the control group will receive usual care at a 6-month follow-up appointment. This will involve a single visit with the GP to discuss the benefits of statin medication to prevent CVD and replicates usual practice.

\section{Outcomes}

\section{Primary}

The primary objective of this study is to test the feasibility of a brief behavioural intervention by a GP with an n-of-1 trial of medication to test adverse events, designed to increase adherence to statin therapy relative to usual care (control). The feasibility study will determine whether to progress to an RCT to test the effectiveness of the openlabel intervention versus usual care. ${ }^{23}$ The following primary outcomes will determine whether to progress to an effectiveness trial:

1. The proportion of invited patients who enrol in the trial.

2. The proportion of enrolled participants who accept the GP offer to engage in a behavioural n-of-1 selfexperimentation.

3 . The proportion of participants in the treatment conditions who decide to continue statin therapy in the open-label arm compared with the proportion who decide to continue statin therapy in the control arm. 


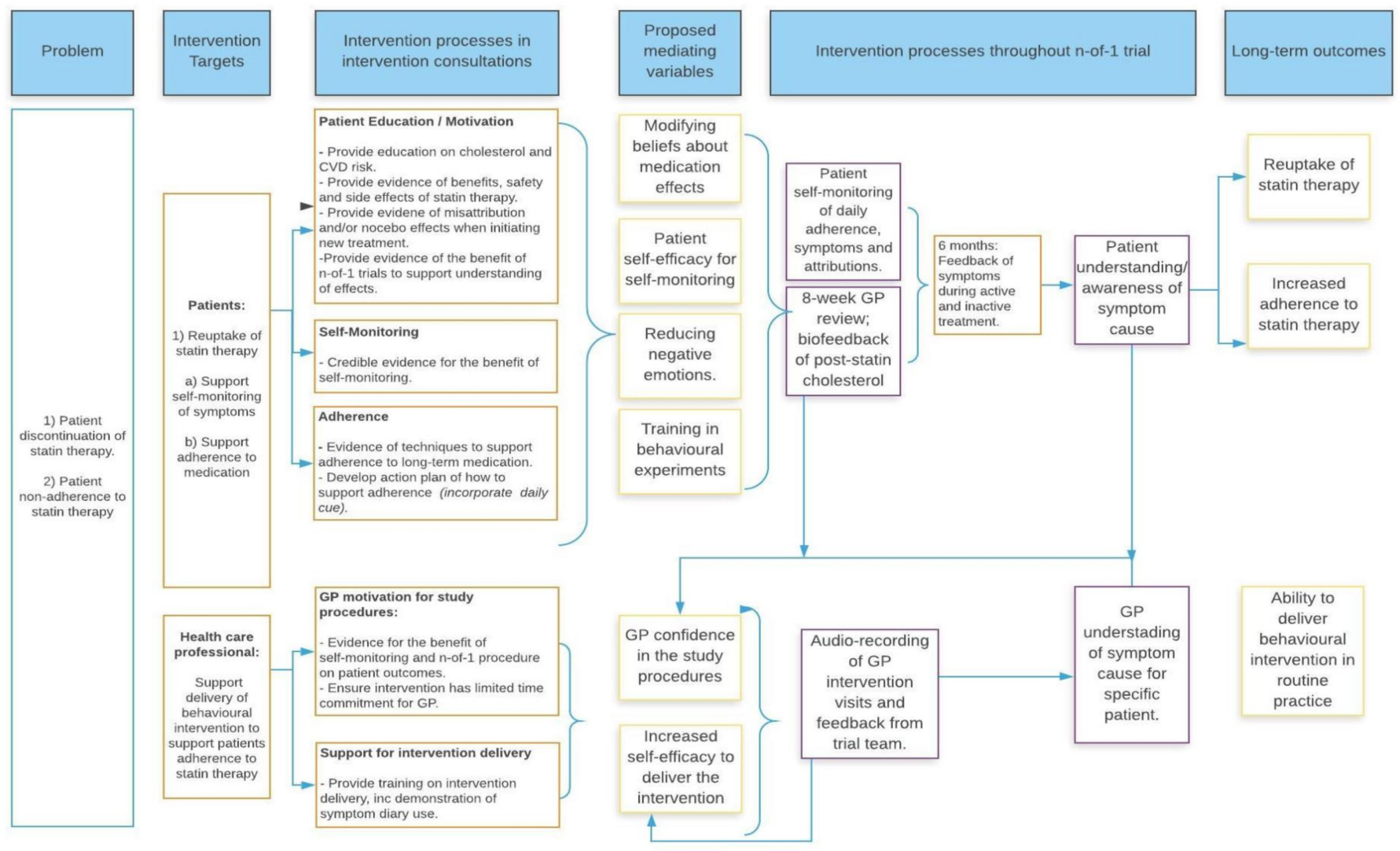

Figure 2 Logic model of intervention development. CVD, cardiovascular disease; GP, general practitioner.

\section{Secondary}

We will combine quantitative and qualitative methods to assess process and effectiveness measures. We have not included some relevant effectiveness measures, such as CVD risk, as the study is not powered to detect these changes. The study will assess measures to (1) determine the most appropriate primary outcome for a future trial, (2) inform sample size estimates for a future trial and (3) aid the further development of the behavioural intervention.

\section{Secondary measures include}

1. The difference in the proportion of participants who decide to continue statin medication 'full time' on the unblinded n-of-1 trial compared with the proportion of participants who decide to continue statin medication in the blinded (positive control) n-of-1 trial.

2. The mean number of self-reported symptoms in the unblinded n-of-1 trial compared with the blinded nof-1 trial.

3 . The count of the number of times that participants attribute side effects to statin medication in the unblinded trial compared with the blinded trial.

4. The difference in mean pain severity scores and mean pain interference scores (measured by the Brief Pain Inventory) between 'active' and 'inactive' treatment periods in the unblinded trial compared with the blinded trial.
5. The difference in mean scores in their beliefs about medication before and after participation in the n-of- 1 trials. The difference in the change in mean scores in beliefs about medication between the unblinded trial and the blinded trial.

\section{Qualitative measures}

1. Participants' acceptance of using alternating medication to better understand their symptoms and intolerance of statin medication.

2. GPs' thoughts about using behavioural interventions to encourage patients to alternate between active and inactive treatment periods in routine practice.

3. If applicable, in the event that many patients decline to participate in the study and the study is unable to recruit the complete sample size, to explore reasons for participants' decision not to participate.

\section{Measurements}

Figure 3 summarises all measurements collected.

\section{Sociodemographic measurements}

Participants will self-report age, sex, highest level of formal education, employment status, ethnicity and postcode at the baseline assessment.

\section{Medical and medication history}

Relevant medical history and current medication. 
Table 2 Summary of behaviour change intervention components, targeted determinants and behaviour change techniques used in the TaSINI study following the behaviour change wheel framework

\section{Intervention component \\ Primary message or resource \\ Intervention function and coded \\ behaviour change techniques}

\section{Brief advice consultations delivered by a GP, facilitated by information leaflet}

1.1 Review of blood cholesterol level and - Explanation of what LDL and HDL discussion of CVD risk.

cholesterol is.

- Review blood test results to indicate to participant what their cholesterol is.

- Explanation of how cholesterol relates to CVD risk. a. Education

- Information about health consequences.

- Information about antecedents.

b. Persuasion

- Information about health consequences.

- Biofeedback.

- Credible source (GP).
1.2 Discussion of physiological effect of statins and motivational advice from GP.

1.3 Discussion of scientific evidence of statin safety and side effects.
Explanation of how statins reduce LDL a. Education cholesterol in the blood.

- Explanation of the extent to which statins reduce CVD risk (reframe taking statins as buying insurance for house).

b. Persuasion

- Information about health consequences.

- Credible source (GP).

- Information about health consequences.

c. Enablement

Framing/ reframing.

a. Education

- Information about health consequences.

- Pros and cons. scientific evidence shows statins are safe

- Provide reassurance that scientific evidence suggests people experience side effects on placebos and statins.

b. Persuasion

- Credible source (GP).

- Information about health consequences.

1.4 Discussion of self-experimentation (n-of-1 trial).
- Explanation of experimentation with medication (ie, n-of-1 trial) with GP support being the only way to know true cause of adverse effects.

- Encourage 'thinking like a scientist' to work out the effects of statin medication.

- Explanation of 'win-win' situation: at the end of the experiment patient will know whether to continue to take statins or not.

Explanation of threat appraisals (ie, the tendency to feel anxious when one experiences symptoms and appraises this to a new medicine) and how to deal with them. a. Education

- Re-attribution.

b. Training

- Behavioural experiments.

- Instructions on how to perform a behaviour.

c. Enablement

- Pharmacological support? (Prompt use/ adherence to a drug to support behaviour change).

- Social support (GP).

- Pros and cons. Problem solving.

- Commitment.

- Reduce negative emotions.

d. Persuasion

- Verbal persuasion about capability.

- Information about emotional consequences.

- Credible source (GP).

- Framing/ reframing

e. Environmental restructuring

- Exposure

a. Enablement - Prompts/cues 
Table 2 Continued

2.2 Participant completion of adherence, - Resource of daily survey to record symptoms and attributions survey. adherence to statin, current symptoms and what the symptoms are attributable to.
a. Training
- Self-monitoring of outcome of behaviour.
- Associative learning.
b. Enablement
- Monitoring of emotional consequences.

\section{Review consultation with GP (8 weeks post intervention).}

\subsection{Review of cholesterol following} 4 weeks of statin medication and discussion of first 8 weeks of $n-o f-1$.

\section{- Show participant updated blood} cholesterol and explain any changes.

- Reiterate benefit of statin medication for CVD risk.

- Troubleshoot any problems participant has experienced in first 8 weeks in preparation for remaining 16 weeks.
a. Education
- Feedback on outcome of behaviour
b. Persuasion
- Biofeedback
- Credible source (GP).
- Problem solving.
c. Incentivisation
- Feedback on outcome of behaviour.
- Biofeedback.

\section{Review consultation with GP (6 months post intervention). \\ 4.1 Feedback daily self-monitoring data. - Show participant overview of adherence, symptom, and attribution data (provided by research team). \\ - Discuss experience of self- experimentation with participant. \\ - Reiterate benefit and safety of statin medication. \\ - Ask participants' decision of whether to resume statin therapy full time.}
a. Education
- Feedback on outcome of behaviour.
b. Persuasion
- Biofeedback.
- Credible source (GP).
- Commitment.

CVD, cardiovascular disease; GP, general practitioner; HDL, High-density lipoprotein; LDL, low-density lipoprotein; TaSINI, Tackling statin intolerance with n-of-1 trials.

\section{Blood sample}

A venous blood sample for lipid profile (high-density lipoprotein, calculated LDL) and total cholesterol) and liver function tests (bilirubin, ALT, AST, ALP, albumin) will be collected prior to the first GP consultation and prior to the 8-week review consultation. Creatine kinase will be measured before the 8-week review consultation.

\section{Questionnaires}

Participants will be asked to complete the Beliefs about Medication Questionnaire (BMQ-General ${ }^{24}$ at the baseline visit with the researcher and after their final follow-up appointment with the GP. The BMQ-General comprises two 4-item factors assessing beliefs about whether medicines are harmful, addictive or overused by doctors.

Participants will be asked to complete a daily questionnaire for the last week of each 4-week treatment blocks. This daily questionnaire will include measures of the following:

1. Adherence, comprising one item: 'Over the last 24 hours, were you able to take your TaSINI study medicine exactly as prescribed?'

2. Symptoms, consisting of four items. Participants are initially asked to 'state the most troublesome symptom you are experiencing today', followed by 'how severe is this symptom today?' $(0=$ no symptoms, $100=$ extremely severe'). These items are repeated for participants to add a second most troublesome symptom, if applicable.

3. Attributions, consisting of one item, 'I believe that the symptom that has been troubling me today is a result of my study medication', answered on a five-point Likert scale (strongly agree to strongly disagree).

4. Brief Pain Inventory (Short Form), comprising 16-items assessing pain severity and interreference over the previous 24 hours period.

\section{Retention and withdrawal}

All participants will be informed of their right to withdraw from the study at any time. If a participant would like to withdraw from the study, a researcher will ask permission for the trial team to use their data collected up to the point at which they have withdrawn from the study. The reason for withdrawal will be recorded in the case report form, along with a note of consent for the use of participant data so far. Participants who are withdrawn will not be replaced. Participants who decide not to accept the GPs offer of the n-of-1 experiment are not considered withdrawn, and will be followed up after 6 months. To promote participant retention and complete the 


\begin{tabular}{|c|c|c|c|c|c|c|c|c|}
\hline & \multicolumn{8}{|c|}{ STUDY VISITS AND DATA COLLECTION POINTS } \\
\hline & $\begin{array}{l}\text { Telephone } \\
\text { screen }\end{array}$ & $\begin{array}{l}\text { Baseline } \\
\text { visit }\end{array}$ & $\begin{array}{l}\text { Blood } \\
\text { test }\end{array}$ & $\begin{array}{l}\text { GP } \\
\text { Intervention }\end{array}$ & $\begin{array}{l}\text { Online } \\
\text { questionnaire } \\
\text { data collection } \\
\text { every day for } \\
\text { last } 7 \text { days of } \\
\text { each } 4 \text { wk } \\
\text { period }\end{array}$ & $\begin{array}{l}\text { Blood } \\
\text { test }\end{array}$ & $\begin{array}{l}\text { Week } \\
8 \mathrm{GP} \\
\text { Visit }\end{array}$ & $\begin{array}{l}6 \\
\text { month } \\
\text { GP Visit }\end{array}$ \\
\hline $\begin{array}{l}\text { Unblinded n-of-1 } \\
\text { intervention }\end{array}$ & $x$ & $\mathrm{x}$ & $x$ & $x$ & $x$ & $\mathrm{x}$ & $x$ & $x$ \\
\hline $\begin{array}{l}\text { Blinded n-of-1 } \\
\text { intervention }\end{array}$ & $\mathrm{x}$ & $x$ & $x$ & $x$ & $x$ & $x$ & $\mathrm{x}$ & $x$ \\
\hline Usual care & $x$ & $x$ & & & & & & $x$ \\
\hline & \multicolumn{8}{|c|}{ PROCEDURES AND ASSESSMENTS } \\
\hline Eligibility assessment & $\mathrm{x}$ & $x$ & & & & & & \\
\hline Informed consent & & $x$ & & & & & & \\
\hline Randomisation & & $x$ & & & & & & \\
\hline Demographics & & $\mathrm{x}$ & & & & & & \\
\hline $\begin{array}{l}\text { Beliefs about Medication } \\
\text { Questionnaire (BMQ) }\end{array}$ & & $x$ & & & & & & $x$ \\
\hline Current Medications & & $x$ & & & & & & \\
\hline $\begin{array}{l}\text { Lab tests (ALT, AST, CK, } \\
\text { lipid profile). }\end{array}$ & & & $x$ & & & $x$ & & \\
\hline $\begin{array}{l}\text { Lipid profile review by } \\
\text { GP }\end{array}$ & & & & $x$ & & & $x$ & \\
\hline Intervention delivery & & & & $x$ & & & & \\
\hline $\begin{array}{l}\text { Adherence to } \\
\text { medication }\end{array}$ & & & & & $x$ & & & \\
\hline $\begin{array}{l}\text { Daily symptom and } \\
\text { attribution }\end{array}$ & & & & & $x$ & & & \\
\hline Brief Pain Inventory & & & & & $x$ & & & \\
\hline $\begin{array}{l}\text { GP records participants } \\
\text { decision re: full time } \\
\text { statin medication. }\end{array}$ & & & & & & & & $x$ \\
\hline
\end{tabular}

Figure 3 Schedule of study visits, procedures and assessments. ALT, alanine aminotransferase; AST, aspartate aminotransferase; CK, creatinine kinase; GP, general practitioner.

follow-up, participants will be offered a $£ 20$ gift card when attending the final GP appointment.

\section{Statistical analysis}

The primary outcome measures for study are progression criteria, and analysis for this will use data from all participants invited and enrolled into the trial. Descriptive and inferential statistics, presenting $95 \%$ CIs will be used to analyse and report the primary outcome measures.

For participants allocated to the intervention arms, we will summarise participants' symptom and attribution data throughout the blinded and unblinded n-of-1 trials, and report this to GPs to discuss in the final consultation with the participant. For each participant, this will comprise $3 \times 7$ days of observations during 'active' treatment (statin medication) and $3 \times 7$ days of observations during 'inactive' treatment (ie, no treatment or placebo medication). For symptom occurrence and attribution, we will give the proportion of days on which the symptom occurred in both 'active' and 'inactive' treatment days and the proportion of days that the patient attributed the symptom to the statin medication. We will not use statistical tests for these.

For presentation to the academic community, we will calculate the mean difference in statin-related symptoms (coded by MedDRA) ${ }^{25}$ for each arm of the trial using generalised linear mixed-effect models using an appropriate link function for either binary or linear data, with the participant set as a random effect. We will calculate the mean differences in daily self-reported pain severity and pain interference for the each of the two treatment arms using generalised mixed-effect models with an appropriate link function for binary or linear data, with participant set as a random effect. The mean differences will also be calculated for BMQ scores for participants before and after the n-of- 1 trials, and between the blinded and unblinded trial arms.

\section{Patient, public and clinician involvement}

At the stage of applying for study funding, we recruited PPI panel members from the Nuffield Department of Primary Care panel who had started medication for a 
long-term condition (or to prevent future disease) that had caused intolerable adverse effects. This advisory panel of five patients informed the intervention design, methods and the development of the intervention materials. This panel will inform the dissemination of the trial results.

At the funding application stage, we also surveyed 211 GPs to explore whether the TaSINI intervention would be appropriate in routine practice. GPs estimated that $37 \%$ of the patients they recommended statin therapy to were concerned about starting statins due to fear of intolerable side effects, and $16 \%$ of patients discontinued the first prescribed statin. Only $6 \%$ of GPs reported using repeated on-off periods to encourage persistence of the offending statin, but $76 \%$ believed the process would be helpful in routine practice. We explained the trial procedures to these GPs and asked whether they would foresee any problems in running such a trial and incorporated the feedback into the intervention development.

\section{Qualitative component}

When the main trial has completed final follow-up visits, we intend to conduct three semistructured focus group interviews with participants; one for participants who enrolled in the trial but did not engage with the n-of-1 experiment, one for participants who started experimenting with statin medication but stopped before the 24-week treatment period was complete and one for participants who completed the n-of-1 intervention. GPs who delivered the TaSINI intervention will be interviewed after the final participant from the site completes the final follow-up visit. We will explore their thoughts about delivering the intervention and how the intervention could be improved for further research. Interviews and focus groups will be recorded, transcribed verbatim and analysed using framework analysis. Framework analysis allows deductive exploration based on the aims and objectives of the interview. A thematic framework for analysis will be constructed prior to the interviews and unanticipated themes arising during the interviews will be added to the framework as appropriate. The qualitative focus groups with patients and interviews with GPs will play a valuable part of the process evaluation of the feasibility trial and inform the development of a largerscale RCT.

\section{Stopping rules}

If, after a significant period of active recruitment, recruitment or engagement with the interventions is not feasible and the recruitment target will not be met, recruitment to the study will be terminated. In this case, we will undertake a qualitative study only with participants who attend a baseline visit with the researcher but who declined to participate and explore their thoughts on what we were proposing. To enact this, we will ask permission to keep the contact details of such patients and to ask to contact them again in the future if necessary.

\section{Ethics and dissemination}

The trial has been prospectively registered on ISRCTN. Modifications of the protocol will be submitted for review by the research ethics committee and amended on the ISRCTN trial registry. If the findings indicate that the intervention is feasible, the results will inform the development and sample size of a larger-scale RCT to test the effectiveness of the intervention on reducing LDL cholesterol. The findings will be submitted to a peer-reviewed journal and may be presented at scientific conferences. On publication, the findings will be made available to participants and to the wider public on the Nuffield Department of Primary Care website.

Acknowledgements The authors would like to thank the members of the TaSINI Patient and Public Involvement Panel from the Nuffield Department of Primary Care Health Sciences for their invaluable input throughout all stages of the study design.

Contributors PA, KT and JH collaborated in designing the study. PA and KT collaborated in obtaining funding for the trial. KT, RF and JB developed the operational aspects of the trial. KT drafted the manuscript. All authors provided critical revisions to the manuscript and read and approved the final manuscript.

Funding The study is funded by the National Institute of Health Research (NIHR) Collaboration for Leadership in Applied Health Research and Care (CLAHRC), Oxford, as well as the NIHR Oxford Biomedical Research Centre (BRC). PA is an NIHR senior investigator and funded by the NIHR Oxford BRC and CLAHRC.

Competing interests None declared.

Patient consent for publication Not required.

Ethics approval The study protocol (V2.0 19.03.2019) was reviewed and approved by the National Research Ethics Service, North of Scotland Research Ethics Service (Ref: 19/NS/0014)

Provenance and peer review Not commissioned; externally peer reviewed.

Open access This is an open access article distributed in accordance with the Creative Commons Attribution Non Commercial (CC BY-NC 4.0) license, which permits others to distribute, remix, adapt, build upon this work non-commercially, and license their derivative works on different terms, provided the original work is properly cited, appropriate credit is given, any changes made indicated, and the use is non-commercial. See: http://creativecommons.org/licenses/by-nc/4.0/.

Author note The trial is sponsored by the University of Oxford, Clinical Trials and Research Governance, Joint Research Office, Block 60, Churchill Hospital, Old Road, Headington, Oxford, OX37LE, UK.

ORCID iD

Paul Aveyard http://orcid.org/0000-0002-1802-4217

\section{REFERENCES}

1 Finegold JA, Manisty $\mathrm{CH}$, Goldacre B, et al. What proportion of symptomatic side effects in patients taking statins are genuinely caused by the drug? systematic review of randomized placebocontrolled trials to aid individual patient choice. Eur J Prev Cardiol 2014;21:464-74.

2 Taylor FC, Huffman M, Ebrahim S. Statin therapy for primary prevention of cardiovascular disease. JAMA 2013;310:2451-2.

3 Armitage $\mathrm{J}$. The safety of statins in clinical practice. The Lancet 2007;370:1781-90

4 Collins R, Reith C, Emberson J, et al. Interpretation of the evidence for the efficacy and safety of statin therapy. The Lancet 2016;388:2532-61.

5 Zhang H, Plutzky J, Skentzos S, et al. Discontinuation of statins in routine care settings: a cohort study. Ann Intern Med 2013;158:526-34.

6 Hoffman KB, Kraus C, Dimbil M, et al. A survey of the FDA's AERS database regarding muscle and tendon adverse events linked to the statin drug class. PLoS One 2012;7:e42866.

7 Matthews A, Herrett E, Gasparrini A, et al. Impact of statin related media coverage on use of statins: interrupted time series analysis with UK primary care data. BMJ 2016;353:i3283-10. 
8 Kashani A, Phillips CO, Foody JM, et al. Risks associated with statin therapy: a systematic overview of randomized clinical trials. Circulation 2006;114:2788-97.

9 National Institute for Health and Care Excellence. Nice guidance draft for consultation lipid modification, 2014. Available: http://www.nice. org.uk/guidance/cg181/documents/lipid-modification-update-draftfull-guideline2

10 Vinogradova Y, Coupland C, Brindle P, et al. Discontinuation and restarting in patients on statin treatment: prospective open cohort study using a primary care database. BMJ 2016;353:i3305.

11 Yusuf S. Why do people not take life-saving medications? the case of statins. The Lancet 2016;388:943-5.

12 Barsky AJ, Saintfort R, Rogers MP. Nonspecific medication side effects and the nocebo phenomenon. JAMA 2002;287:622-7.

13 Vohra S, Shamseer L, Sampson M, et al. Consort extension for reporting n-of-1 trials (cent) 2015 statement. J Clin Epidemiol 2016;76:9-17.

14 Joy TR, Monjed A, Zou GY, et al. N-Of-1 (single-patient) trials for statin-related myalgia. Ann Intern Med 2014;160:301-310-10.

15 Herrett E, Williamson E, Beaumont D, et al. Study protocol for statin web-based investigation of side effects (StatinWISE): a series of randomised controlled $\mathrm{n}$-of- 1 trials comparing atorvastatin and placebo in UK primary care. BMJ Open 2017;7:e016604-2.

16 Mihaylova B, Emberson J, Blackwell L, et al. The effects of lowering LDL cholesterol with statin therapy in people at low risk of vascular disease: meta-analysis of individual data from 27 randomised trials. Lancet 2012;380:581-90.
17 Wei MY, Ito MK, Cohen JD, et al. Predictors of statin adherence, switching, and discontinuation in the usage survey: understanding the use of statins in America and gaps in patient education. $J$ Clin Lipidol 2013;7:472-83

18 Korhonen MJ, Pentti J, Hartikainen J, et al. Somatic symptoms of anxiety and nonadherence to statin therapy. Int $J$ Cardiol 2016;214:493-9.

19 Wouters H, Van Dijk L, Geers HCJ, et al. Understanding statin NonAdherence: knowing which perceptions and experiences matter to different patients. PLoS One 2016;11:e0146272.

20 Yardley L, Ainsworth B, Arden-Close E, et al. The person-based approach to enhancing the acceptability and feasibility of interventions. Pilot Feasibility Stud 2015;1:1-7.

21 Michie S, Atkins L, West R. The behaviour change wheel: a guide to designing intervention. London: Silverback Publishing, 2014.

22 Michie S, Johnston M, Abraham C, et al. Making psychological theory useful for implementing evidence based practice: a consensus approach. Qual Saf Health Care 2005;14:26-33.

23 Gartlehner G, Hansen R, Nissman D. Criteria for distinguishing effectiveness from efficacy trials in systematic reviews. 12. Rockville (MD: Agency for Healthcare Research and Quality (US), 2006.

24 Horne R, Weinman J, Hankins M. The beliefs about medicines questionnaire: the development and evaluation of a new method for assessing the cognitive representation of medication. Psychol Health 1999;14:1-24.

25 Medical dictionary for regulatory activities. Available: https://www. meddra.org [Accessed 14 Jul 2019]. 